\title{
HUBBLE SPACE TELESCOPE STIS SPECTRUM OF RW AURIGAE A: EVIDENCE FOR AN IONIZED BELTLIKE STRUCTURE AND MASS EJECTION IN TIMESCALES OF A FEW HOURS
}

\author{
Ana I. Gómez de Castro \\ Instituto de Astronomía y Geodesia (CSIC-UCM), Universidad Complutense de Madrid, 28040 Madrid, Spain; \\ aig@mat.ucm.es \\ AND \\ Eva VERDUGo \\ ISO Data Centre, VILSPA, P.O. Box 50727, 28080 Madrid, Spain; \\ everdugo@iso.vilspa.esa.es \\ Received 2003 January 31; accepted 2003 July 11
}

\begin{abstract}
Profiles of the UV semiforbidden lines of C III $]_{1908}$ and Si III $]_{1892}$ of RW Aur have been obtained with the Hubble Space Telescope (HST) STIS. The C III] ${ }_{1908}$ profile shows two high-velocity components at $v= \pm 170$ $\mathrm{km} \mathrm{s}^{-1}$ and a central one. The Si III $]_{1892}$ profile is very broad $\left(\mathrm{FWHM}=293 \mathrm{~km} \mathrm{~s}^{-1}\right)$, and the high-velocity components are unresolved. The high-velocity components are most probably produced in a rotating belt similar to that detected in other sources of bipolar outflows. A radius between $2.7 R_{*}$ and the corotation radius $\left(6.1 R_{*}\right)$ is derived, and a $\log T_{e}(\mathrm{~K}) \simeq 4.7$ and $\log n_{e}\left(\mathrm{~cm}^{-3}\right)=11.6$ are estimated. The belt is clumpy, and the most likely source of heating is local X-ray radiation, probably associated with the release of magnetic energy. In addition, profiles of the optical lines of $\mathrm{He}$ I, Fe II, and $\mathrm{H} \alpha$ retrieved from the $H S T$ archive have been analyzed. The spectra were obtained by shifting the STIS slit between exposures in the transverse direction to the flow. Two features vary from one exposure to another: a blueshifted emission feature (detected in the $\mathrm{Fe}$ II and $\mathrm{He}$ I lines) and a redshifted absorption feature (detected in $\mathrm{H} \alpha$ ), which are observed at the velocity of the blueshifted and redshifted components of the jet, respectively. There is a clear-cut correlation between the equivalent widths of these two components. These components are not associated with the flaring activity of the source, since they remain stable timescales of $\simeq 40$ minutes. They are most likely associated with variations in the stellar/circumstellar environment in timescales of a few $(\simeq 5)$ hours, consistent with reports by other authors for variations of the veiling and the appearance of shell signatures in the optical spectrum.
\end{abstract}

Subject headings: stars: individual (RW Aurigae) — stars: pre-main-sequence — ultraviolet: stars

\section{INTRODUCTION}

Determining the precise physical mechanism that connects accretion to outflow is one of the key issues in star formation. The T Tauri phase is the most interesting for this purpose since, at this stage, bipolar flows - often detected as optical jets - are still active, but the central engine can be observed at optical and ultraviolet wavelengths, allowing a detailed spectroscopic diagnosis. The most suitable spectral indicators are in the ultraviolet (UV) range, where the semiforbidden intercombination lines of abundant species such as $\mathrm{O}$ III, Si III, and C III are observed. These lines are optically thin and strong in the spectra of the T Tauri stars (TTSs; Gómez de Castro \& Franqueira 1997). The hot plasma $\left(T_{e} \simeq 5 \times 10^{4} \mathrm{~K}\right)$ traced by these lines is expected to be detected in association with the accretion flow and the stellar atmosphere. Moreover, recent UV observations have shown that there is also a contribution from a hot jet (Gómez de Castro \& Verdugo 2002, 2001).

In this work, we analyze two sets (UV and optical) of Hubble Space Telescope (HST) spectroscopic data of RW Aurigae A, a TTS that has been extensively studied, so the main physical parameters are well known (see Table 1). Also, the spectral signatures of the jet (Dougados et al. 2000; Hirth et al. 1994; Hirth, Mundt, \& Solf 1997; Haman 1994), accretion flow (Petrov et al. 2001, hereafter P2001), and the stellar atmosphere (P2001) are well known. In $\S 2$, both data sets (UV and optical HST STIS mid-resolution spectra) are described. The UV data consist of profiles of the $\mathrm{C}$ III $]_{1908}$ and Si III $]_{1892}$ lines. The seven optical stellar spectra were obtained during a time lapse of $12 \mathrm{hr}$, as a byproduct of an observing campaign intended to detect rotation signatures in the jet. The data are analyzed in $\S 3$, and the major results are summarized in $\S 4$. A broad emission component is detected and resolved in the $\left.\mathrm{C}_{\mathrm{III}}\right]_{1908}$ profile into two components at $\pm 170 \mathrm{~km} \mathrm{~s}^{-1}$. In $\S 5$, the possible connection of this spectroscopic component with a corotating envelope is discussed. A density of $\log n_{e}\left(\mathrm{~cm}^{-3}\right)=11.6$ and a radius between 2.7 and $6.1 R_{*}$ are derived. The major conclusions of this work are summarized in $\S 6$.

\section{OBSERVATIONS}

Optical and ultraviolet spectra of RW Aur obtained with the HST STIS are analyzed. The UV data correspond to our program, intended to determine the physical conditions at the base of the optical jet. The optical data have been retrieved from the archive.

\subsection{Ultraviolet Data}

The HST STIS data were obtained with the midresolution grating $\mathrm{G} 230 \mathrm{M}(R \sim 10,500)$ and the MAMA detector (see Table 2). The observations were carried out on 
TABLE 1

Physical Characteristics of RW Aur A ${ }^{\mathrm{a}}$

\begin{tabular}{|c|c|c|}
\hline Characteristic & Value & Source \\
\hline \multirow{2}{*}{ 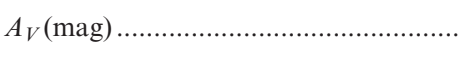 } & $<0.3$ & 1 \\
\hline & $<0.7$ & 2 \\
\hline Spectral Type. & K4 & 3 \\
\hline Mass (g) . & $2.19 \times 10^{33}$ & 2 \\
\hline $\operatorname{Radius}\left(R_{\odot}\right)$. & $1.3-1.5$ & 2 \\
\hline$V_{r}$ & $16.0 \pm 6$ & 4 \\
\hline 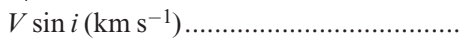 & $16-40$ & 2 \\
\hline Equator inclination $(\mathrm{deg}) \ldots \ldots \ldots \ldots \ldots \ldots \ldots$ & $\geq 46 \pm 4$ & 5 \\
\hline \multirow{2}{*}{ 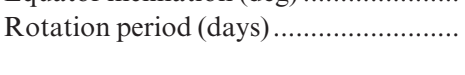 } & 5.4 & 6 \\
\hline & 3.2 & 7 \\
\hline
\end{tabular}

a A distance of $140 \mathrm{pc}$, which is the distance from the TaurusAuriga molecular cloud, has been assumed. The parallax derived from Hipparcos is very uncertain: $14.18 \pm 6.8$ mas.

References.-1. Ghez, White, \& Simon 1997. 2. Petrov et al. 2001. 3. Stout-Batalha et al. 2000 4. Gahm et al. 1999. 5. López-Martín et al. 2003. 6. Grinin, Petrov, \& Shakhovskaya 1983. 7. Calculated from $V \sin (i)$ and $R_{*}=1.4 R_{\odot}$.

2000 September 16 . The $52^{\prime \prime} \times 0$ ". 2 slit was used, and the position angle was 218.77 , nearly perpendicular to the jet. The spatial scale is $0.029 \mathrm{pix}^{-1}$. The emitting region is unresolved. The spectrum covers the 1840-1930 A range. The Si III $]_{1892}$ and $\left.\mathrm{C} \mathrm{III]}\right]_{1908}$ intercombination lines are clearly detected: the forbidden components ( $\mathrm{Si}$ III $]_{1803}$ and $\mathrm{C}$ III $]_{1907}$ ) are not. The total exposure time was $2222 \mathrm{~s}$. The exposure time was split into three subexposures of roughly $740 \mathrm{~s}$ each, which have been analyzed independently to search for spectral variability. Large variations that could be associated with flare activity have not been detected. The $\mathrm{C}$ IIII $]_{1908}$ and Si III $]_{1892}$ co-added profiles are plotted in Figure 1.

\subsection{Optical Data}

Long-slit spectroscopy of RW Aur was carried out in the optical range (6245-6915 ^) on 2000 December 10 (Woitas et al. 2002). The seven long-slit spectra were obtained to map the circumstellar environment and the jet: the slit was always oriented parallel to the jet axis, and the center was shifted perpendicularly to the jet by $-0.21,-0$ ". $14,-0$ ". 07 , $0^{\prime \prime}, 0.07,0.14$, and 0.21 from the stellar position. The $52^{\prime \prime} \times 0$ ".2 slit was used. A summary of the main characteristics of the observations is shown in Figure 2 (see also Table 2).
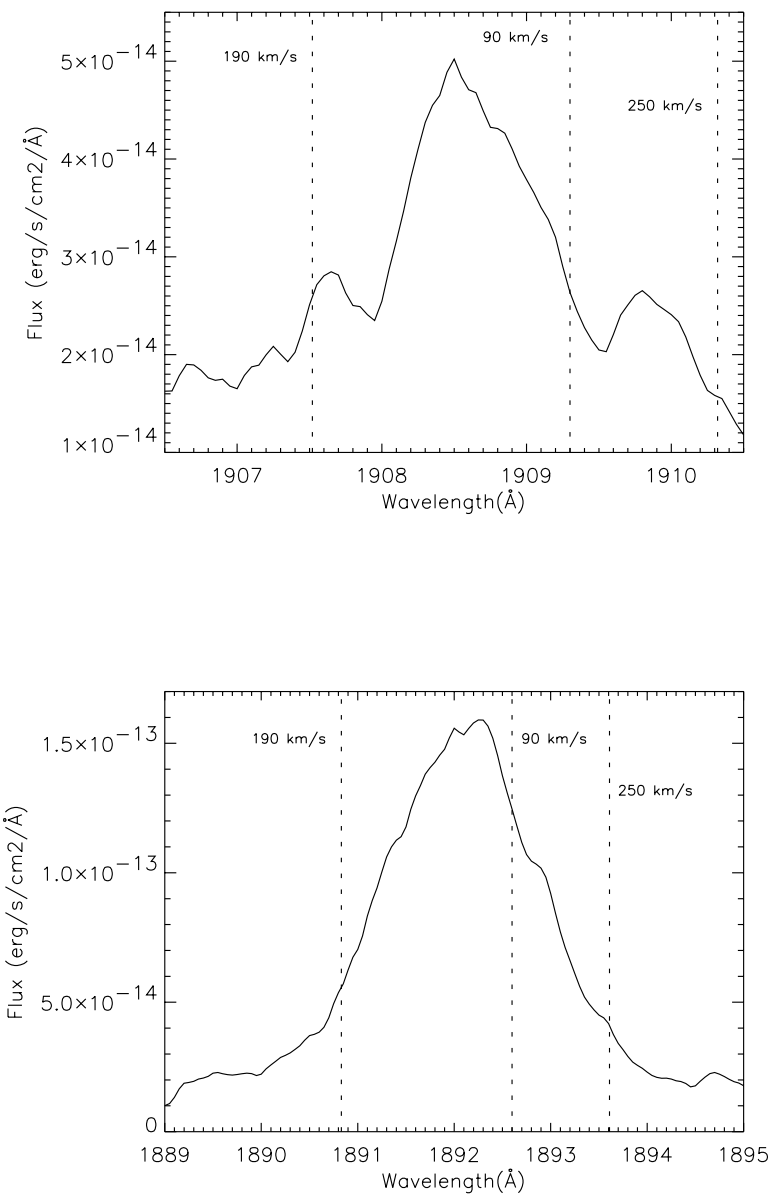

FIG. 1.-Top: $\mathrm{C}$ III] $]_{1908}$ co-added profile. Velocity of the red and blue components of the jet is marked, as well as the accretion flow component at $250 \mathrm{~km} \mathrm{~s}^{-1}$. Bottom: Si III] $]_{1892}$ co-added profile. Jet and accretion flow components are marked as in the top panel.

The distribution of circumstellar matter in the jet direction is not symmetric. The luminosity distribution along the slit shows (in all the spectra) that the scattering material extends further in the direction toward the redshifted flow. In particular, at offset $-0 . " 21$, two peaks are observed at 0 ".14 and 0 " 10 from the stellar position for the redshifted and blueshifted parts of the flow, respectively. The distance between the two peaks is 0.24 , ruling out the possibility of

TABLE 2

HST OBSERVATIONS (STIS) OF RW Aur A

\begin{tabular}{|c|c|c|c|c|c|c|c|c|}
\hline $\begin{array}{c}\text { Date of } \\
\text { Observation }\end{array}$ & GMT & Mode & $\begin{array}{c}\text { Spectral } \\
\text { Resolution }\end{array}$ & $\begin{array}{l}\text { Wavelength } \\
\text { Range } \\
\text { (A) }\end{array}$ & $\begin{array}{l}T_{\exp } \\
\text { (s) }\end{array}$ & $\begin{array}{c}\text { Position Angle } \\
\text { of the Slit }\end{array}$ & $\begin{array}{l}\text { Offset }^{\mathrm{a}} \\
(\operatorname{arcsec})\end{array}$ & Obs. ID \\
\hline 2000 Sep $16 .$. & $14: 59: 31$ & G230M & 10500.0 & $1839-1929$ & 2222.00 & 218.77 & 0.00 & O62N03010 \\
\hline 2000 Dec $10 \ldots$ & $08: 25: 04$ & G750M & 5980.00 & $6295-6867$ & 2328.00 & 129.65 & 0.14 & O5DG01010 \\
\hline 2000 Dec $10 \ldots$ & $10: 09: 33$ & G750M & 5980.00 & $6295-6867$ & 2202.00 & 129.65 & 0.00 & O5DG01020 \\
\hline 2000 Dec $10 \ldots \ldots \ldots \ldots . . . . .$. & $11: 38: 00$ & G750M & 5980.00 & $6295-6867$ & 2592.00 & 129.65 & -0.14 & O5DG01030 \\
\hline $2000 \operatorname{Dec} 10 \ldots \ldots \ldots \ldots \ldots$ & $13: 27: 10$ & G750M & 5980.00 & $6295-6867$ & 2328.00 & 129.65 & 0.21 & O5DG02010 \\
\hline 2000 Dec $10 \ldots \ldots \ldots \ldots \ldots$ & $14: 58: 59$ & G750M & 5980.00 & $6295-6867$ & 2202.00 & 129.65 & 0.07 & O5DG02020 \\
\hline 2000 Dec $10 \ldots \ldots \ldots \ldots \ldots$ & $16: 27: 26$ & G750M & 5980.00 & $6295-6867$ & 2160.00 & 129.65 & -0.07 & O5DG02030 \\
\hline 2000 Dec $10 \ldots \ldots \ldots \ldots \ldots$ & 17:09:08 & G750M & 5980.00 & $6295-6867$ & 2562.00 & 129.65 & -0.21 & O5DG02040 \\
\hline
\end{tabular}

\footnotetext{
a Offset from the stellar position perpendicular to the slit orientation.
} 
a) $\operatorname{Pos}=0 \operatorname{arcsec}$

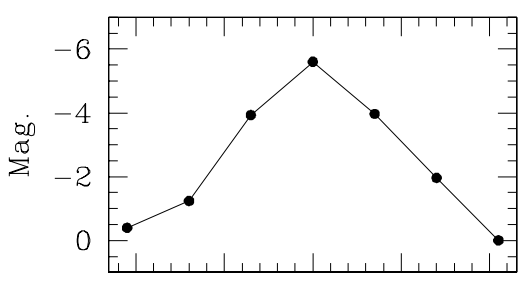

b) $\operatorname{Pos}=1.44$ l.d.
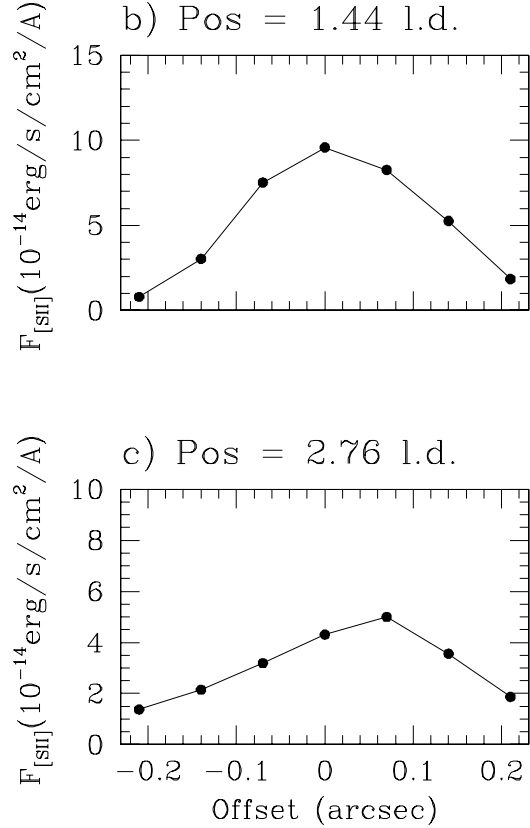

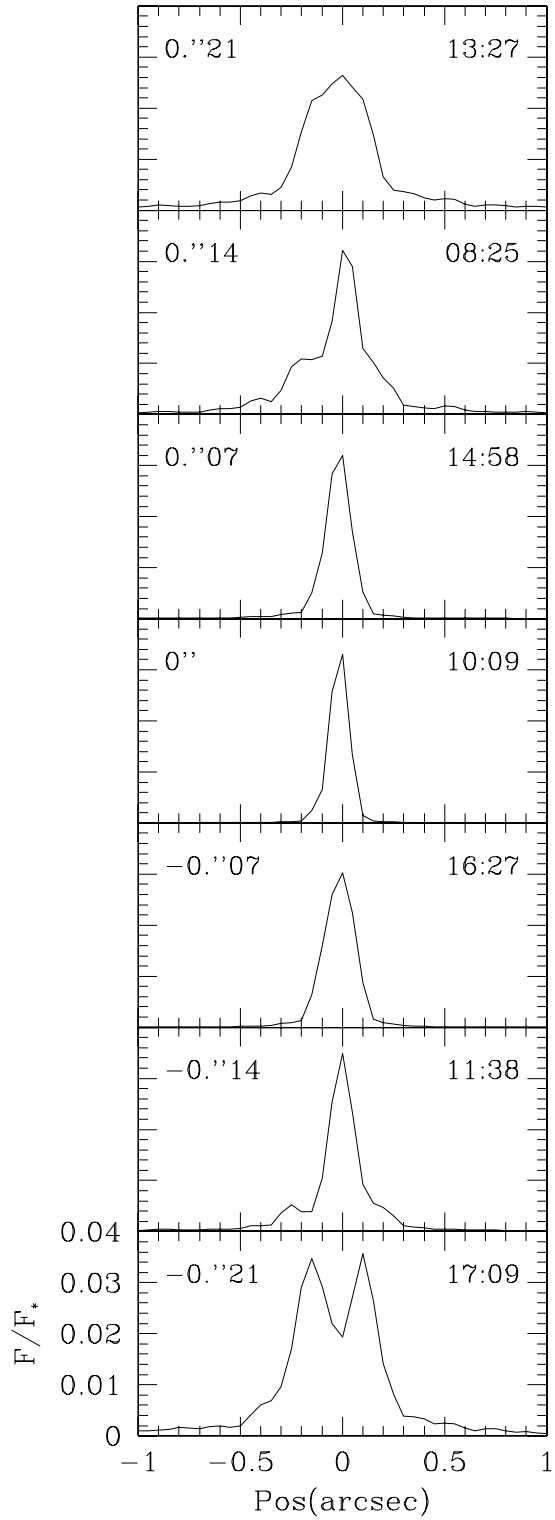

FIG. 2.- Summary of the optical mapping of RW Aur carried out with HST STIS, showing flux distribution perpendicular to the jet (left) and along the slit (right). Slit width is 0 ". 2 (28 AU or 0.16 light days). Flux variation across the jet has been measured at the stellar position and at two knots situated at 1.44 and 2.76 light days from the star. Top: Flux variation is plotted for the stellar continuum in a logarithmic (magnitudes) scale relative to the minimum flux. Middle and lower panels: $[\mathrm{S} \mathrm{II}]$ lines flux coming from the knots represented in a linear scale. Right column: Variation of the spatial distribution of the continuum flux along the slit. At offset of $-29.4 \mathrm{AU}\left(-00^{\prime \prime} 21\right)$, the absorption by the circumstellar disk is clearly apparent.

an instrumental effect caused by the brightness of the source. ${ }^{1}$

Figure 2 is also suggestive of an anisotropy of the luminosity distribution perpendicular to the jet direction (e.g., in the offset direction): the star seems to be brighter at positive than at negative offsets. This apparent asymmetry is most probably an observational effect caused by the observing strategy. The data were obtained in two sequences. First, the star was

\footnotetext{
1 The diffraction pattern of the star is $\mathrm{X}$-shaped, and it is possible that as the slit is shifted over the target, different parts of the $\mathrm{X}$ pattern are sampled. This could produce two ghost stellar spectra, e.g., a doublepeaked structure in the luminosity distribution along the slit symmetric with respect to the stellar position (J. Valenti 2002, private communication). The ghost spectra should be at \pm 0 ".21 for an slit offset of $-00^{\prime \prime} 21$, so the separation between the two peaks should be 0."42. Such a doublepeaked structure should also be observed for an slit offset of +0 ". 21
}

centered in the slit, ${ }^{2}$ and then the observations at offsets 0.21 , $0^{\prime \prime}$, and -0 ". 21 were obtained. Then the star was again centered in the slit, and a new sequence at offsets $0.14,0.07$, -0 ". 07 , and -0 ". 14 was obtained. HST has a drift rate of less $^{3}$ than milliarcseconds per hour; therefore, the real offset from the stellar position is larger at the end of each sequence than what could produce the observed asymmetry.

The velocities of the blue- and redshifted components of the jet are $-192 \pm 5$ and $90 \pm 4 \mathrm{~km} \mathrm{~s}^{-1}$, respectively (see Woitas et al. 2002 for a detailed analysis of the data). The velocities have been measured with respect to the stellar rest

\footnotetext{
${ }^{2}$ For the ACQ/peak-up procedure, see the HST STIS Reference Manual.

${ }^{3}$ Go to http://www.stsci.edu/hst/stis/strategies/targetacq / documents/handbooks/currentIHB/c08_acq2.html\#445823.
} 
frame determined from the Li I $]_{6708}$ absorption line and are similar to those reported from earlier observations (Hirth et al. 1994, 1997; Haman 1994), although a slight shift to blueward velocities during the last $10 \mathrm{yr}$ is noticeable from -165 to $-190 \mathrm{~km} \mathrm{~s}^{-1}$ in the blueshifted component of the jet and from 110 to $90 \mathrm{~km} \mathrm{~s}^{-1}$ in the redshifted component.

The stellar spectrum has been extracted by co-adding the four central rows; i.e., the peak of the luminosity distribution along the slit has been determined, and we have used an extraction slit of 4 pixels' height. As a consequence, only a $70 \%$ of the energy is encircled within the extraction size, ${ }^{4}$ but source confusion is minimized. The FHWM of the stellar spectrum is 3.3 pixels or 0 ".17 (in agreement with the STIS first-order spatial profile for grid $\mathrm{G} 750 \mathrm{M}$ ) and dominates any possible contribution from the nebula.

The dominant features in the stellar spectrum are the absorption lines of Si II $(6349,6373 \AA)$, the emission lines of He I (6678.15 $\AA$ ), Li I, and $\mathrm{H} \alpha$, as well as three strong emis- sion features at 6432.65 and $6516 \AA$ corresponding to $\mathrm{Fe}$ II[40] and at $6456.4 \AA$ corresponding to $\mathrm{Fe}$ II[74]. ${ }^{5}$ The Si II absorption lines are slightly redshifted, in agreement with P2001 data. Both lines correspond to the same multiplet. In optically thin plasma, the $6349 \AA$ line should be twice as strong as the $6373 \AA$ line. However, they have similar strengths, which illustrates the high optical thickness of the line. No significant variations are detected in any of the absorption lines. The emission features of Fe II, He I, and $\mathrm{H} \alpha$ are illustrated in Figure 3. The occasional presence of a redshifted absorption component is clearly apparent in the $\mathrm{H} \alpha$ profile. At an offset of \pm 0 ". 07 , the absorption is maximum: the red wing of $\mathrm{H} \alpha$ is significantly absorbed. At offsets of \pm 0 ". 14 and $-00^{\prime \prime} 21$, the $\mathrm{H} \alpha$ absorption is minimal, and at offset 0.21 , it increases again. A variable blueshifted component is clearly detected in the Fe II and He I emission lines. We have not detected variations of the spectrum along the slit.

\footnotetext{
${ }^{4}$ See the HST STIS Handbook or go to http://www.stsci.edu/hst/stis.
}

${ }^{5}$ All the optical wavelengths in this work are air wavelengths.
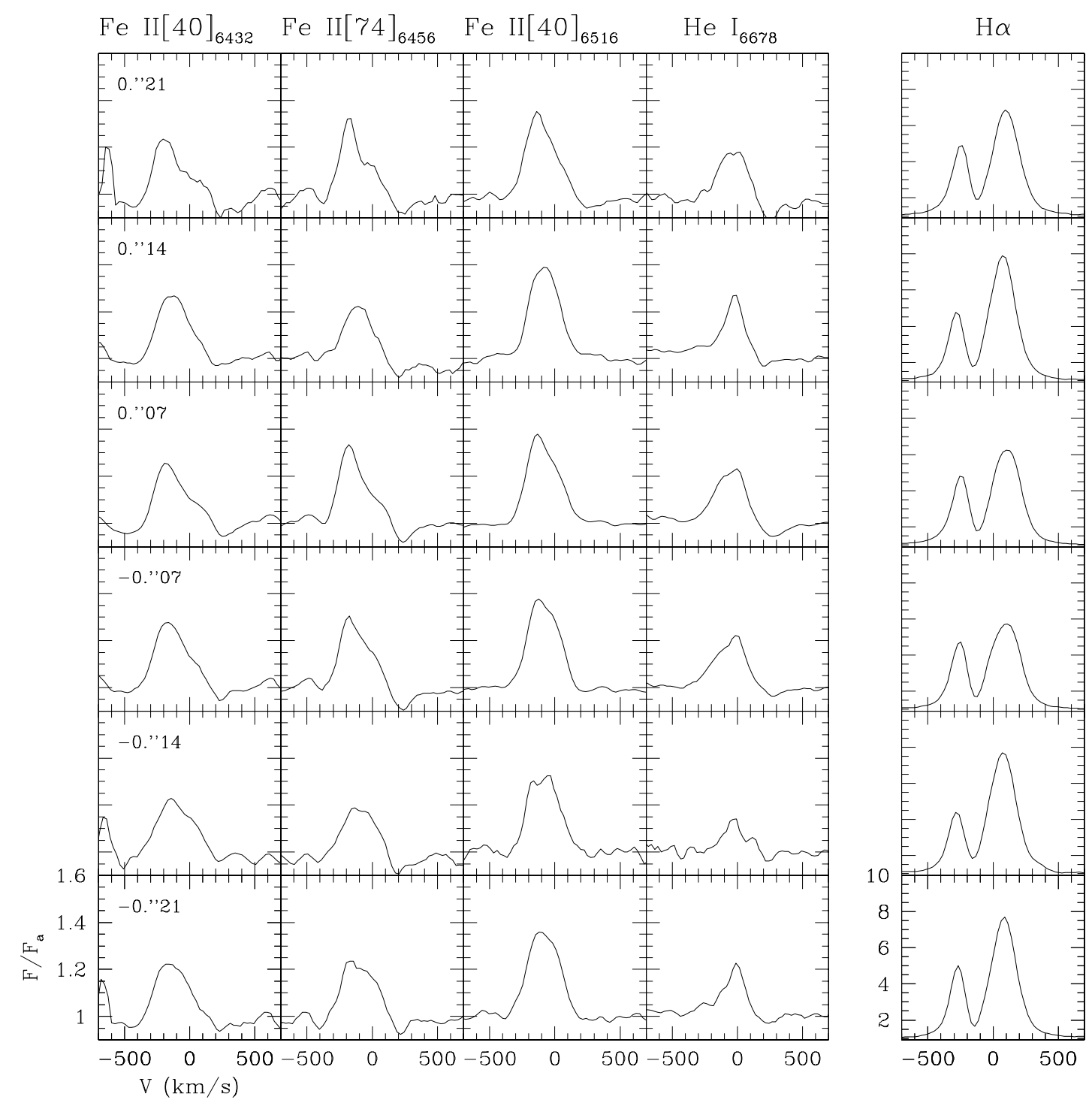

FIG. 3.-Profiles of the main emission lines detected in the optical spectrum of RW Aur. Notice that the profiles change from one observation to another. The major changes are observed in the $\mathrm{Fe}$ II[74], $\mathrm{He} \mathrm{I}$, and $\mathrm{H} \alpha$ lines. 


\section{DATA ANALYSIS}

\subsection{Interpretation of the Variations Detected in the Optical Spectra}

The optical profiles of some spectral lines change from one slit position to another. At some locations, a blueshifted emission component is observed only in some spectral lines and is accompanied by the presence of a redshifted absorption component only in $\mathrm{H} \alpha$. This effect cannot be caused by spurious wavelength shifts associated with an uneven illumination of the slit, since the velocity shift is $\geq 100 \mathrm{~km} \mathrm{~s}^{-1}$, while the miscentering of the object in the slit could account for $30 \mathrm{~km} \mathrm{~s}^{-1}$ as much. ${ }^{6}$ Therefore, it is associated with a real physical component that for conciseness we call the highvelocity component (HVC). As RW Aur is surrounded by a reflection nebula, both components could contribute in a different manner to the observed spectrum, depending on the slit location. The nebular scattering profile can be derived from the observed continuum flux contribution perpendicular to the jet. If we focus the discussion in the most accurately known offsets $\left(0^{\prime \prime}, 0\right.$, 07,0 ". 14 , and 0 ".21; see Fig. 3 ), it is apparent that the observations are best fit by an exponential law with a characteristic $e$-folding spatial scale of $\simeq 40$ mas or $5.7 \mathrm{AU}^{7}$. Note that this scale represents an upper limit to the true one, since the data correspond to the convolution between the true spatial distribution of the scattered radiation and the slit transmittance. This small $e$ folding spatial scale is consistent with a small dust column toward the source, as pointed out independently by the rather low extinction of RW Aur (see Table 1) and the small polarization (Bastien 1982). In this context, the difference between spectra at offsets 0 ."07 and 0".14 (see Fig. 3) could be interpreted as a result of the presence of some obscuring material in the light path from the region where the HVC is formed and the region sampled at offset 0 ". 14 , which does not affect the stellar radiation. This obscuring screen should be located very close to the star because

1. The spectrum at offset 0 ". 14 is dominated by the scattered stellar radiation coming from the innermost region within the 0 ".20 slit, roughly from $r \sim 0$ ". $04\left(0\right.$ " $\left.14-00^{\prime \prime} 10\right)$, given the exponential profile described above.

2. The spectrum at offset 0.21 is very similar to the spectrum at offset 0. . 07 , so the light paths of the stellar and the $\mathrm{HVC}$ radiation must be very similar. If the light path of the HVC was significantly larger, the relative strength of the $\mathrm{HVC}$ to the low-velocity component of the Fe II and $\mathrm{He}$ I lines should vary accordingly. Moreover, extra $\mathrm{H} \alpha$ absorption should be detected.

Therefore, a very peculiar (and highly unlikely) geometry should be invoked so the observations can be fit in this framework.

Another interesting possibility is that the blue- and redshifted components are associated with frequent flaring activity. In such a case, as the flaring characteristic timescale in T Tauri stars is $\simeq 300-1000$ s (Glassgold, Feigelson, \& Montemerle 2000), the blue- and redshifted components

\footnotetext{
${ }^{6}$ See the HST STIS Handbook or go to http://www.stsci.edu/hst/stis.

${ }^{7} F_{\Delta \lambda}(r)=F_{\Delta \lambda, 0} \exp -[r(\mathrm{mas}) /(40 \pm 1)]$, where $F(r)$ is the stellar continuum flux measured in a featureless window and $r$ is the offset in milliarcseconds (mas). If, for instance, $\Delta \lambda$ corresponds to the featureless 6610-6630 A range, then $F_{\Delta \lambda, 0}=(4.6 \pm 0.4) 10^{-10} \mathrm{ergs}^{-1} \mathrm{~cm}^{-2}$ and the correlation coefficient between $F(r)$ and $r$ is 0.99897 .
}

would be detected only when, by chance, a flare occurs during the observation. We have been able to test this possibility, since each spectra was split into six subexposures to remove cosmic rays. The exposure time of the subexposures is $\simeq 400 \mathrm{~s}$, which is comparable to the flaring timescale allowing flare detection. As shown in Figure 4 for offset

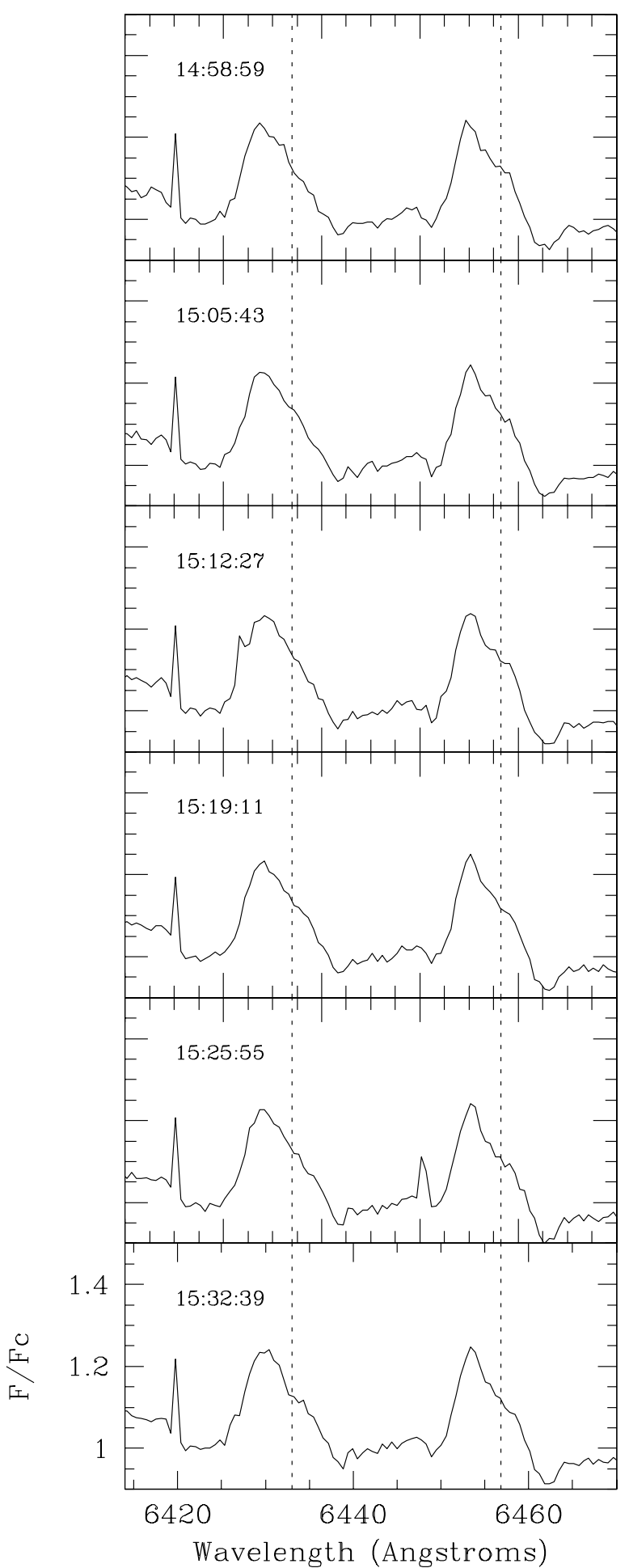

FIG. 4.-Profiles of the Fe II[74] lines corresponding to the observation with O5DG02020. Top to bottom: Six subexposures from GMT $14^{\mathrm{h}} 58^{\mathrm{m}} 59^{\mathrm{s}}$ to $15^{\mathrm{h}} 32^{\mathrm{m}} 39^{\mathrm{s}}$. Exposure times are $360 \mathrm{~s}$, except for the last subexposure (bottom), which has an exposure time of $402 \mathrm{~s}$. 
$0^{\prime \prime} 07$, no significant variations are detected among the subexposures. Hence, the blue- and redshifted components are not associated with flaring and are stable over timescales of $\simeq 2200$ s.

We conclude that the most likely interpretation of the variations found in the spectra is that the stellar spectrum has changed between the first sequence of the observations (when the spectrum at offset 0 ". 14 was obtained) and the second sequence of observations (when the spectra at offsets 0 ".21 and $0 . .07$ were obtained). This points out a variability timescale of around $5 \mathrm{hr}$. The light traveling time between 0 ".07 (9.8 AU) and 0 ".21(29.4 AU) is $2.7 \mathrm{hr}$, which is consistent with the fact that both regions display similar spectral characteristics. In fact, RW Aur is known to be variable in timescales of just a few hours (see Stout-Batalha, Batalha, \& Basri 2000; P2001).

\subsection{Variability}

Variability is best tracked in the $\mathrm{Fe}$ II[74], $\mathrm{He} \mathrm{I}$, and $\mathrm{H} \alpha$ lines (see Fig. 3). There is a variable blueshifted emission component in the $\mathrm{Fe}$ II [74] and He I lines, and the redshifted component of $\mathrm{H} \alpha$ is also significantly variable. The equivalent widths have been measured for the $\mathrm{Fe}$ II[74] and $\mathrm{He}$ I lines and for the redshifted component of $\mathrm{H} \alpha$ (see Table 3). As shown in Figure 5, they are strongly correlated. The correlation coefficients of $W(\mathrm{H} \alpha)$ and $W(\mathrm{He} \mathrm{I})$ with $W(\mathrm{Fe}$ II $)$ are 0.92 and 0.98 , respectively. ${ }^{8}$ The light curves are represented in Figure 6 using the GMT of the observation as reference.

To further study the characteristics of the variable component, a quiescent profile has been defined. For the Fe II [74] and the $\mathrm{He} \mathrm{I}$ lines, this is calculated as the average of the spectra taken at offsets \pm 19.6 AU to improve the signal-tonoise ratio $(\mathrm{S} / \mathrm{N})$. The quiescent profile has been substrated out from the observed profiles, and the results are plotted in Figure 7. The variable blueshifted emission component is clearly sorted out. This component is observed at $-204 \mathrm{~km}$ $\mathrm{s}^{-1}$ in the Fe II[74] line and at $-145 \mathrm{~km} \mathrm{~s}^{-1}$ in the He I line, where the $\mathrm{S} / \mathrm{N}$ is worse and self-absorption effects are important (see Fig. 7 in P2001).

\footnotetext{
${ }^{8}$ The least-squares fits are $W(\mathrm{H} \alpha)=(-14.7 \pm 3.3), W(\mathrm{Fe}$ II $)+(56.7 \pm$ $4.8)$, and $W(\mathrm{He} \mathrm{I})=(1.38 \pm 0.14) W(\mathrm{Fe} \mathrm{II})-(1.0 \pm 0.2)$.
}

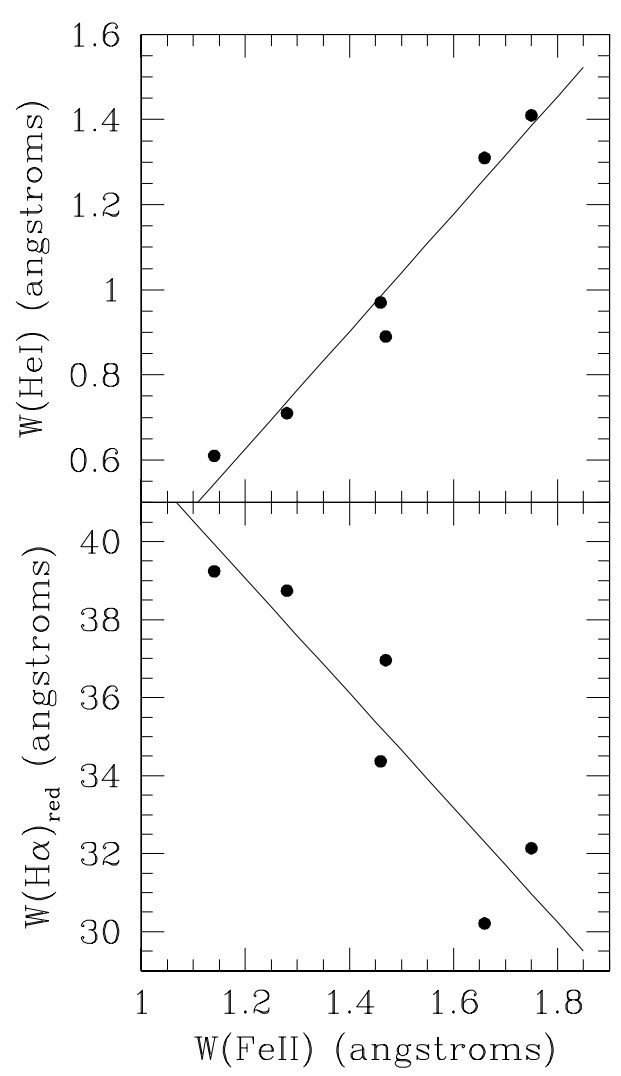

FIG. 5.-Top: Correlation between the variability of the equivalent width of the $\mathrm{Fe}$ II [74], $W(\mathrm{Fe}$ II), and the He I, W(He I) lines. Bottom: Correlation between the variability of $W(\mathrm{Fe}$ II $)$ and the equivalent width of the red component of the $\mathrm{H} \alpha$ profile, $W(\mathrm{H} \alpha)$.

The analysis of the $\mathrm{H} \alpha$ profile variations is more complex: the strength and the high optical thickness of the line produce very broad wings that are especially sensitive to the spectral centering between the observed and the quiescent profile. As the $\mathrm{S} / \mathrm{N}$ is good enough, only one spectrum (the corresponding to offset $-19.6 \mathrm{AU}$ ) has been used to represent the quiescent state. Then the $\mathrm{H} \alpha$ profiles have been centered carefully by means of a cross-correlation analysis. After that, the quiescent $\mathrm{H} \alpha$ profile has been subtracted from the rest. The resulting profiles are also plotted in Figure 7: the velocity of the blue- and redshifted components of

TABLE 3

Variability of the Optical Stellar Spectra

\begin{tabular}{|c|c|c|c|c|c|}
\hline$i$ & $\begin{array}{l}\text { Slit Offset } \\
\quad(\mathrm{AU})\end{array}$ & $\begin{array}{c}\text { GMT } \\
\text { (hr) }\end{array}$ & $\begin{array}{c}W(\mathrm{Fe} \mathrm{II}[74]) \\
(\AA)\end{array}$ & $\begin{array}{c}W(\mathrm{He} \mathrm{I}) \\
(\AA)\end{array}$ & $\begin{array}{c}W(\mathrm{H} \alpha)_{r}{ }^{\mathrm{a}} \\
(\AA)\end{array}$ \\
\hline $1 \ldots \ldots$ & -29.4 & 17.15 & 1.47 & 0.89 & 36.96 \\
\hline $2 \ldots \ldots \ldots \ldots \ldots \ldots \ldots$ & -19.6 & 11.63 & 1.14 & 0.61 & 39.24 \\
\hline 3 ............................ & -9.8 & 16.46 & 1.66 & 1.31 & 30.21 \\
\hline $4 \ldots \ldots \ldots \ldots \ldots \ldots \ldots \ldots$ & 0 & 10.16 & $\mathrm{~b}$ & $\mathrm{~b}$ & $\mathrm{~b}$ \\
\hline $5 \ldots \ldots \ldots \ldots \ldots \ldots \ldots \ldots \ldots \ldots \ldots \ldots \ldots$ & 9.8 & 14.98 & 1.75 & 1.41 & 32.13 \\
\hline 6............................ & 19.6 & 08.42 & 1.28 & 0.71 & 38.74 \\
\hline 7 & 29.4 & 13.45 & 1.46 & 0.97 & 34.37 \\
\hline
\end{tabular}

a Only the equivalent width of the red component of the line is indicated.

${ }^{\mathrm{b}} \mathrm{H} \alpha$ was saturated in this spectrum. In addition, the strong interferential fringes make difficult the precise determination of the continuum, so $W(\mathrm{Fe}$ II $)$ and $W(\mathrm{He} \mathrm{I})$ are not measured. 


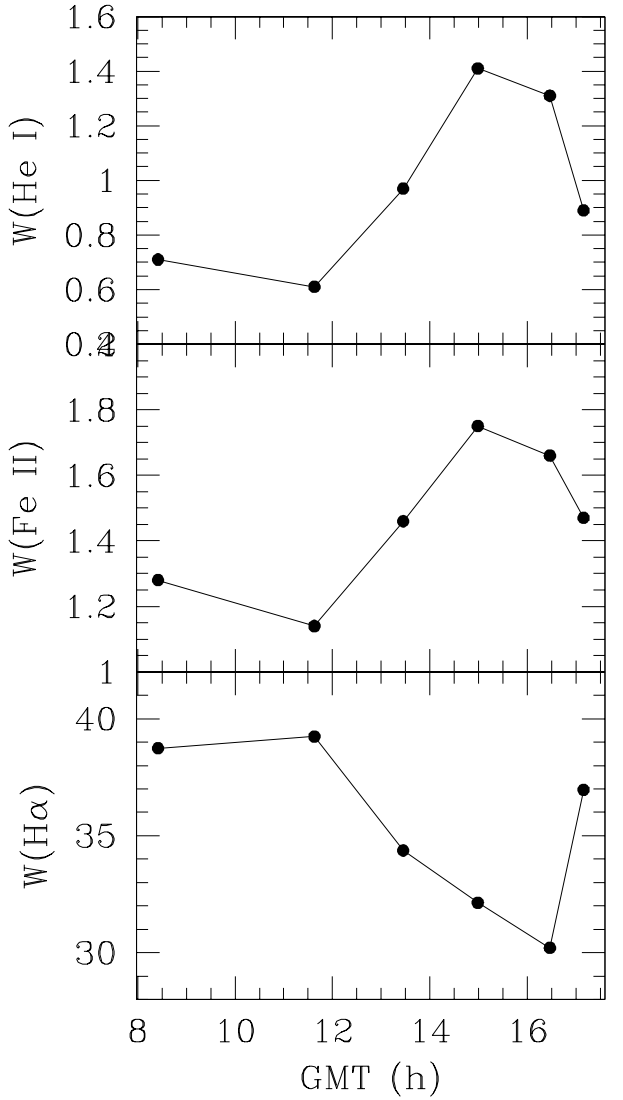

FIg. 6.-Light curves illustrating the variations of the equivalent widths of the $\mathrm{He}$ I, $\mathrm{Fe}$ II[74], and the red component of the $\mathrm{H} \alpha$ line.

the optical jet is marked. As shown in the figure, there is a prominent absorption component at $\simeq 100 \mathrm{~km} \mathrm{~s}^{-1}$. This velocity is significantly smaller than the velocity of the accretion flow, which is $250 \mathrm{~km} \mathrm{~s}^{-1}$ and has remained constant during the last $10 \mathrm{yr}$ (see the high spectral resolution and high S/N optical spectra of RW Aur [P2001]; Beristain, Edwards, \& Kwan 2001). In addition, some weak emission components are detected in the $\mathrm{H} \alpha$ profiles. They are produced by small changes the morphology of the blue and red peaks of the $\mathrm{H} \alpha$ profile (see also Fig. 3). High-resolution profiles of the $\mathrm{H} \alpha$ (and also the $\mathrm{H} \beta$ ) lines should be obtained to analyze in detail these components and determine the possible role of line opacity effects.

\subsection{Comparison between the UV and Optical Quiescent Profiles}

The physical conditions traced by the $\mathrm{He}$ I line and the UV lines are similar: the excitation potential of the He I line is high $\left(\chi_{\mathrm{exc}}=20.8 \mathrm{eV}\right)$ and temperatures between $3 \times 10^{4}$ and $8 \times 10^{4} \mathrm{~K}$ are required to produce line emission in collisionally excited plasmas. As shown in Figure 8, the He I quiescent profile consists of a central emission component and an absorption component redshifted by $250 \mathrm{~km} \mathrm{~s}^{-1}$, e.g., the velocity of the accretion flow. The central emission has a FWHM of $137 \mathrm{~km} \mathrm{~s}^{-1}$, and hence, it is significantly narrower than the $\mathrm{Si} \mathrm{III}]_{1892}$ profile. Its FWHM is like the width of the central component of the $\mathrm{C}$ III $]_{1908}$ profile. No absorption component can be observed in the FUV lines, since they are semiforbidden, although there might be some contribution from the accretion flow to the red wing of the Si III $]_{1892}$ profile (see Fig. 8, bottom).

The Fe II lines trace very different physical conditions from the FUV lines, since their excitation potentials are around $3 \mathrm{eV}$. In Figure 9, the quiescent profile of $\mathrm{Fe}$ II [74] is plotted. Notice that the profile is very asymmetric but it is not an inverse P-Cygni profile. The profiles of the metallic lines of RW Aur are often interpreted in terms of two components: broad and narrow (see P2001; Haman \& Persson 1992). The broad component has a FWHM of 200-280 km $\mathrm{s}^{-1}$ in the $\mathrm{Fe} \mathrm{I}$ and $\mathrm{Fe}$ II lines and seems to be rather stationary (see Figs. 4 and 9 in P2001). The narrow component is variable and may be observed in emission (blue- or redshifted) or as a centered absorption. They have a FWHM of $\simeq 40 \mathrm{~km} \mathrm{~s}^{-1}$, which is not resolved in the HST spectra. In this sense, the asymmetric quiescent profile of the $\mathrm{Fe}$ II lines observed during the HST run is rather peculiar; although occasionally a similar behavior has been detected (see P2001, their Fig. 4, top). We think that the most reasonable source of this asymmetry is the overlap of the redshifted absorption component caused by the accretion flow onto the broad, symmetric profile with a FWHM of around 300 $\mathrm{km} \mathrm{s}^{-1}$, as sketched in Figure 9.

\subsection{UV Data: On the Source of the Si III $]_{1892}$ Line Broadening}

The $\mathrm{C}$ III $]_{1908}$ co-added profile has three clear components centered at $168,-27$, and $-170 \mathrm{~km} \mathrm{~s}^{-1}$ (see Table 4). The central component is most likely caused by the stellar atmosphere, although the slight blueshift suggests that there might be a nonnegligible contribution from a hot wind (see also Fig. 1). The source of the blue- and redshifted components is unclear.

The co-added $\mathrm{Si}$ III $]_{1892}$ profile is very broad, with a FWHM of $1.85 \AA\left(285 \mathrm{~km} \mathrm{~s}^{-1}\right)$ compared with the central component of the $\mathrm{C}$ III $]_{1908}$ line, which has a FWHM of 0.96 $\AA\left(151 \mathrm{~km} \mathrm{~s}^{-1}\right) .{ }^{9}$ This broadening cannot be caused by stellar rotation $\left(v \sin i=16 \mathrm{~km} \mathrm{~s}^{-1} ; \mathrm{P} 2001\right)$ and is unusual in the TTSs (Gómez de Castro \& Verdugo 2002). It seems most

\footnotetext{
${ }^{9}$ It is also broader than the FWHM of the whole C III] $]_{1908}$ line when a single Gaussian fitting to the three resolved components is carried out $\left(\mathrm{FWHM}=1.50 \AA=235 \mathrm{~km} \mathrm{~s}^{-1}\right)$.
}

TABLE 4

Si III AND C III COMPONENTS

\begin{tabular}{|c|c|c|c|c|c|c|c|}
\hline Species & $\begin{array}{c}v_{r} \\
\left(\mathrm{~km} \mathrm{~s}^{-1}\right)\end{array}$ & $\begin{array}{c}F_{r} \\
\left(\mathrm{ergs} \mathrm{s}^{-1} \mathrm{~cm}^{-2}\right)\end{array}$ & $\begin{array}{c}v_{0} \\
\left(\mathrm{~km} \mathrm{~s}^{-1}\right)\end{array}$ & $\begin{array}{c}F_{0} \\
\left(\mathrm{ergs} \mathrm{s}^{-1} \mathrm{~cm}^{-2}\right)\end{array}$ & $\begin{array}{l}\mathrm{FWHM}_{0} \\
\left(\mathrm{~km} \mathrm{~s}^{-1}\right)\end{array}$ & $\begin{array}{c}v_{b} \\
\left(\mathrm{~km} \mathrm{~s}^{-1}\right)\end{array}$ & $\begin{array}{c}F_{b} \\
\left(\operatorname{ergs~s}^{-1} \mathrm{~cm}^{-2}\right)\end{array}$ \\
\hline Si III]. & 170 & $2.24 \times 10^{-14}$ & -3 & $2.37 \times 10^{-13}$ & 235 & -181 & $2.66 \times 10^{-14}$ \\
\hline 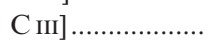 & 168 & $0.69 \times 10^{-14}$ & -27 & $0.41 \times 10^{-13}$ & 151 & -170 & $0.73 \times 10^{-14}$ \\
\hline
\end{tabular}



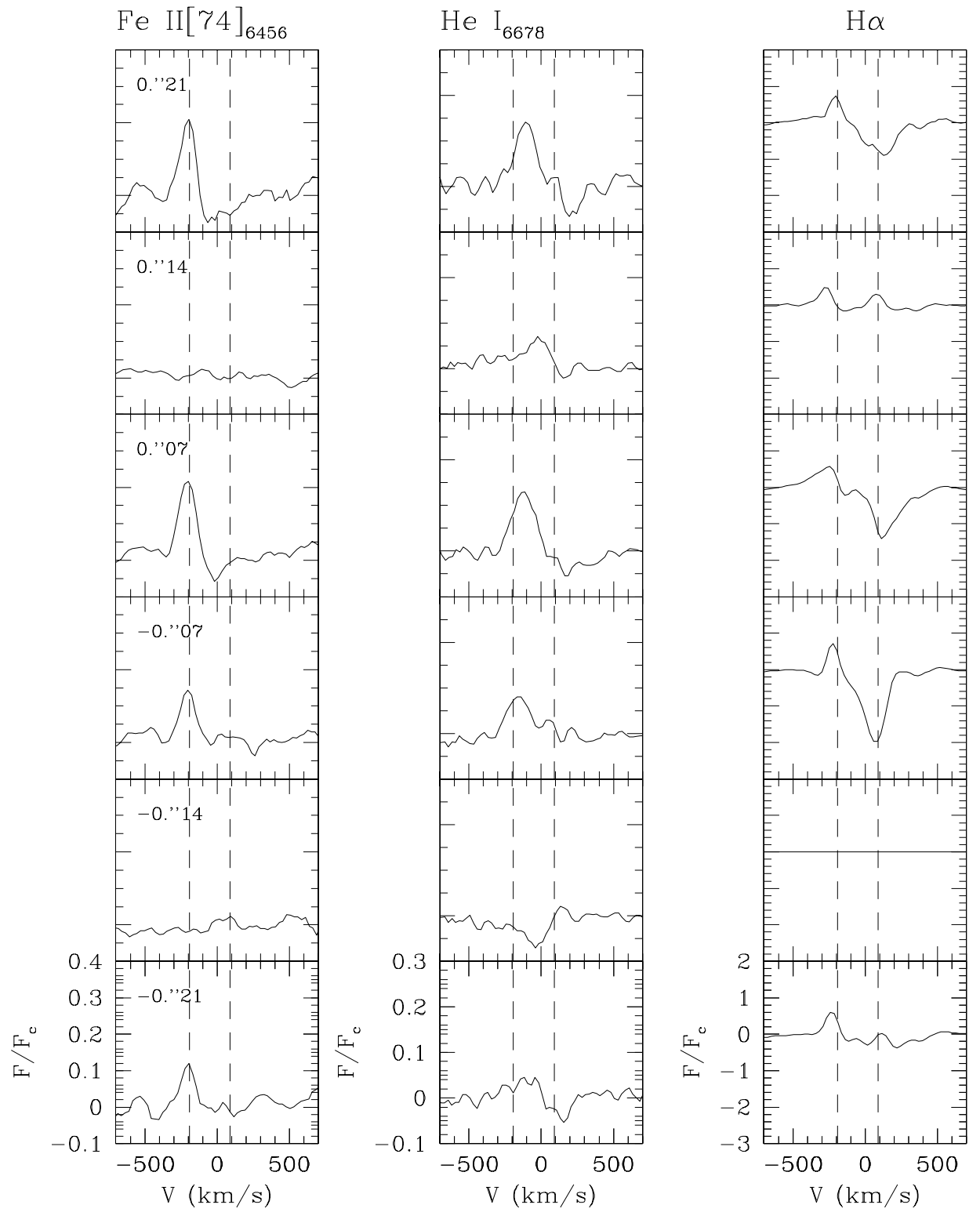

FIG. 7.-Profiles of the variable component of the Fe II[74], He I, and $\mathrm{H} \alpha$ lines. Quiescent profiles have been subtracted out from the individual profiles. Dashed lines: Velocity of the blueshifted and redshifted optical jets.

likely that this huge broadening is caused by the contribution of the two HVCs detected in the $\mathrm{C}$ III $]_{1908}$ line. Notice that although both ions have similar atomic structures, the C III $]_{1908}$ line becomes collisionally quenched at high electron densities, and hence, the Si III $]_{1892}$ line becomes stronger than the C III] $]_{1908}$ line. This is probably because the HVCs are unresolved in the $\mathrm{Si}$ IIII $]_{1892}$ profile. If the $\left.\mathrm{Si} \mathrm{III]}\right]_{1892}$ line is fit to three Gaussians, two HVCs at 170 and $-181 \mathrm{~km} \mathrm{~s}^{-1}$ are required to reproduce the profile (see Table 4).

\section{SUMMARY OF RESULTS FROM THE OBSERVATIONS}

From the analysis of the observations, the following conclusions can be put forward:

1. There are two variable features in the profiles of the optical lines: (a) A variable blueshifted emission component (VBEC), which is detected in the $\mathrm{Fe}$ II and $\mathrm{He} \mathrm{I}$ lines. The velocity of this component is like the velocity of the blueshifted component of the jet.

(b) A variable redshifted absorption component (VRAC), which is detected in $\mathrm{H} \alpha$. The velocity of this component is like the velocity of the redshifted component of the jet, and it is significantly smaller than the velocity of the accretion flow.

2. There is a clear-cut correlation between the equivalent widths of the VBEC and the VRAC. The interpretation of this correlation hangs on the interpretation of the source of the VRAC.

(a) If the VRAC is associated with the redshifted jet, this correlation indicates that mass ejection occurs simultaneously in the red- and blueshifted components of the jet. A similar interpretation was given by Johns \& Basri (1995) 


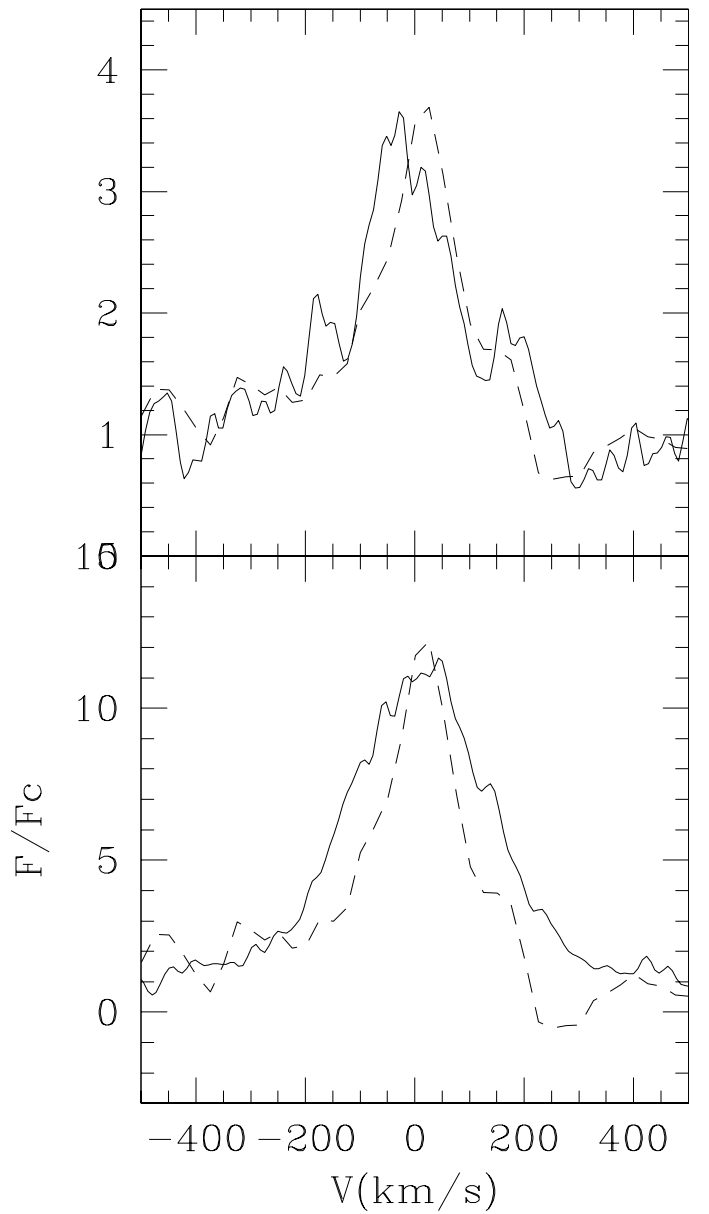

FIG. 8.-Quiescent profile of the He I line (dashed line) is overplotted on the C III $]_{1908}$ (top) and Si III $]_{1892}$ (bottom) profiles. The scale of the He I profile has been adjusted so the peak emission has the same strength as the peak emission of the FUV lines.

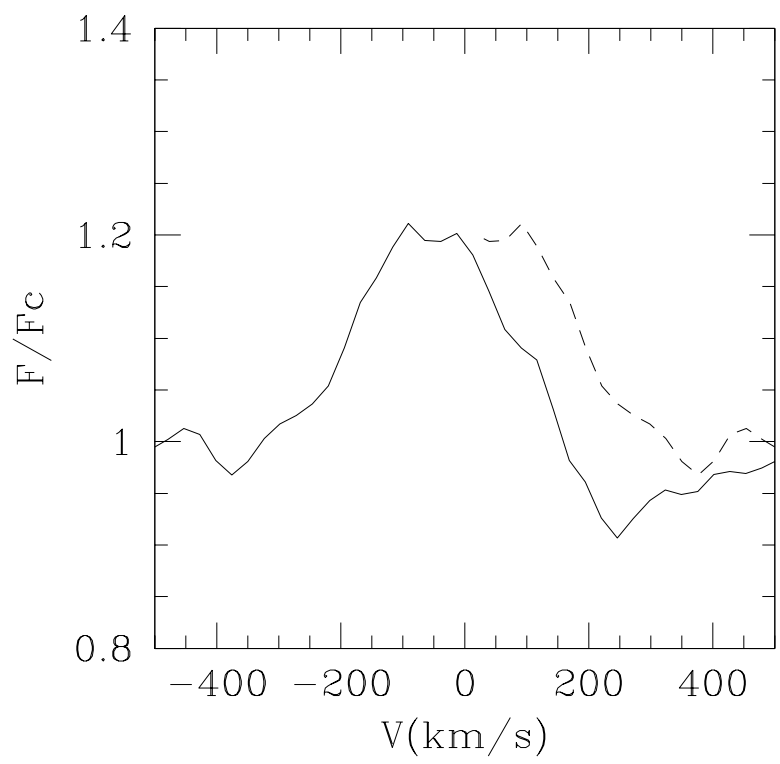

FIG. 9.-Quiescent profile of the Fe II[74] line is plotted. Dashed line: Sketch of the probably symmetric underlying profile, which would be observed in the absence of redshifted absorption. from the time series analysis of the $\mathrm{H} \alpha$ variations. However, it is puzzling that $\mathrm{H} \alpha$ absorption is detected in connection with the redshifted jet instead of emission: this suggests that the density of the red-and blueshifted jet is not the same, which is rather unusual.

(b) If the VRAC is associated with the accretion flow, then this correlation indicates that accretion and ejection events take place simultaneously. However, the velocity of the VRAC is $\sim 150 \mathrm{~km} \mathrm{~s}^{-1}$ smaller than the velocity of the accretion flow.

Therefore, no definite conclusion of the meaning of this correlation can be reached without a short timescale $(\sim 5$ hr), high spectral-resolution monitoring of the $\mathrm{H} \alpha$ and $\mathrm{H} \beta$ variations that allow precise identification of the kinematical features and detection of line opacity effects.

3. The timescale of the variations is larger than a few minutes, demonstrating a lack of association with the usual flaring activity detected in the $\mathrm{T}$ Tauri stars.

4. The timescale of the variations is of few $(\sim 5)$ hours, which is consistent with the timescales reported for the variation of the rotation velocity (P2001) and the equivalent width of the $\mathrm{Li}$ I, $\mathrm{Ca} \mathrm{I}$, or the $\mathrm{Ti}$ I lines (Stout-Batalha et al. 2000), which are best explained by the presence of a warm absorbing shell. This $\sim 5 \mathrm{hr}$ timescale is similar to the free-fall timescale, suggesting a connection between the variable features and the accretion process.

5. There are three quiescent components contributing to the line profiles:

(a) A redshifted component produced by the accretion flow, which is observed in absorption in the permitted optical lines (He I and also, probably, as the source of the asymmetry of the Fe II lines). It could have a minor contribution to the red wing of the profile of the Si III $]_{1892}$ semiforbidden emission line.

(b) A broad emission component centered at the stellar velocity. This component is clearly observed in the UV Si III $]_{1892}$ line, and it has been often observed in the optical profiles of the metallic lines (P2001); in fact, it is most probably underlying the Fe II optical lines presented in this work. The low $\mathrm{S} / \mathrm{N}$ of the He I line, which is dominated by a narrow central emission, precludes its detection in the HST data.

The broad component is resolved into two HVCs at $v= \pm 170 \mathrm{~km} \mathrm{~s}^{-1}$ in the $\left.\mathrm{C} \mathrm{IIII}\right]_{1908}$ profile. The large broadening of the Si III $]_{1892}$ profile can be produced by the presence of these two HVCs. The profiles of the optical metallic lines are also compatible with this interpretation (see P2001, Fig. 4, bottom).

(c) A narrow emission component at rest (or slightly redshifted) with respect to the star, which is clearly observed in the He I line. This component could contribute to the profiles of the FUV lines, e.g., to the central component of the C III $]_{1908}$ line, but it is unresolved.

\section{DISCUSSION: THE PHYSICAL SOURCE OF THE BROAD EMISSION COMPONENT (BEC)}

From the data, it is clear that the redshifted and blueshifted components detected in the $\mathrm{C} \mathrm{III}]_{1908}$ profile, the broadening of the optical metallic lines (P2001), and the Si III] $]_{1892}$ line cannot be ascribed to any of the well-known spectroscopic components of the RW Aur A circumstellar 
environment, e.g. , blueshifted and redshifted jets, star, or accretion flow. Notice that the kinematics of the jet does not seem to change significantly from the large scale (Woitas et al. 2002) to the small scale (López-Martín, Cabrit, \& Dougados 2003). Also, the inclination of the circumstellar disk (see Table 1) would produce a different extinction of the red with respect to the blue component of the jet, which is not observed, since both have similar strengths.

The high spectral-resolution optical profiles show clearly that the BEC broadening is non-Gaussian. Neither can stellar rotation be the source of this broadening, as assessed in $\S 3.4$. Therefore, the emission has to be produced in a structure (shell, belt, etc.) rotating around the star. Belts and rotating envelopes have been detected in many different sources of bipolar outflows (from planetary nebulae to active galactic nuclei). Also, a corotating envelope has been detected around the magnetically active young star AB Dor (Brandt et al. 2001; Gómez de Castro 2002). Some assumptions on the rotation properties of the belt have to be made to derive its radius. Its high temperature provides some clues, since it must be in a region where energy is damped into heating and therefore must be close to the star. Two extreme values can be derived, assuming that the belt is either corotating with the star (e.g., associated to the stellar magnetosphere) or in Keplerian orbit (e.g., controlled by the stellar gravitational field). If corotating,

$$
R_{\text {belt }}=\frac{170 \mathrm{~km} \mathrm{~s}^{-1}}{V \sin (i)} R_{*},
$$

where $V \sin i$ represents the projected rotation velocity of the star. ${ }^{10}$ As indicated in Table $1, V \sin i$ ranges from 16 to $30 \mathrm{~km} \mathrm{~s}^{-1}$. The most often quoted values are 18 (Gahm et al. 2001) to $22 \mathrm{~km} \mathrm{~s}^{-1}$ (Stout-Batalha et al. 2000). The largest values seem to be associated with the occasional presence of a shell that modifies the profiles of the atmospheric lines (P2001). From these values, we derive that $R_{\text {belt }}$ is between 7.7 and $8.8 R_{*}$. These values are far too large, since the inner border of the accretion disk, which is usually assumed to be accretion disk-star corotation radius, is at $6.1 R_{*}$. Otherwise, if we assume that the belt is in Keplerian orbit, we derive a radius of $2.7 R_{*}$, which is very close to the star. We conclude that the belt radius is between 2.7 and 6.1 $R_{*}$, probably rotating at an intermediate velocity, which is controlled by the interaction between the stellar magnetosphere and the accretion disk. This beltlike structure is most likely the source of the optical veiling and could be formed in the equatorial plasma sheet as a consequence of the accretion process. The reported variations in the veiling (P2001; Stout-Batalha et al. 2000) are most likely associated with fluctuations in this structure.

The density of the belt can be constrained from the $\mathrm{Si}$ III/C III ratio. The C III] $]_{1908}$ flux has been derived directly from the data to be $1.1 \times 10^{-14}$ ergs $\mathrm{s}^{-1} \mathrm{~cm}^{-2}$ $\left(F_{\text {blue }}=0.73 \times 10^{-14}\right.$ and $F_{\text {red }}=0.69 \times 10^{-14} \mathrm{ergs} \mathrm{s}^{-1}$ $\mathrm{cm}^{-2}$ ). The large broadening of the $\mathrm{Si} \mathrm{III}_{1892}$ profile is assumed to be caused by the contribution of the belt. From the fit, we derive that the $\mathrm{Si}$ III $]_{1892}$ flux of the blue- and redshifted components are $2.66 \times 10^{-14}$ and $2.24 \times 10^{-14} \mathrm{ergs}$ $\mathrm{s}^{-1} \mathrm{~cm}^{-2}$, respectively. The detection of $\left.\mathrm{O} \mathrm{III}\right]_{1661,1666}$

\footnotetext{
${ }^{10}$ Assuming that the rotation axis of the belt is aligned with the rotation axis of the star.
}

(Gómez de Castro \& Lamzin 1999) can be used to fix up the temperature, since the $\mathrm{O}$ III/Si III ratio is very sensitive to it. If the gas is assumed to be in coronal equilibrium, we derive a $\log T_{e} \simeq 4.7$, which implies that $n_{e} \simeq 4 \times 10^{11} \mathrm{~cm}^{-3}$. The total emitting volume can be calculated from the line strength: ${ }^{11} \quad V=0.31 \times 10^{30} \mathrm{~cm}^{3}$ (and $M=3.7 \times 10^{17} \mathrm{~g}$ ). This emitting volume is very small, ${ }^{12}$ suggesting that the filling factor of the emitting gas is small. Therefore, the belt is expected to consist of filaments and clumps.

This clumpy nature, together with the broad temperature range covered by the various spectral tracers (from $\log T_{e} \simeq 4.7$ traced by the FUV $\operatorname{lines}$ to $\log T_{e} \simeq 3.6$ by the optical metallic lines; P2001), suggest that the excitation mechanism could be photoionization instead of collisional excitation. To further investigate whether the density diagnosis based on the FUV lines is significantly affected by photoionization, we have run some calculations making use of CLOUDY (Ferland 1996), a code designed to simulate emission line regions in astrophysical environments. Two basic models have been explored: photoionization of a dense belt by the ambient X-ray field around the TTSs and photoionization of small clumps of gas around reconnecting magnetic loops. The major assumptions and results are as follows:

Photoionization by the ambient radiation field.-There are numerous sources of X-ray radiation in the circumstellar environment of the TTSs: stellar corona, X-ray radiation at the interface disk-magnetosphere, plasma instabilities producing reconnection events, etc. The X-ray energy distribution of the TTSs is often modeled by two components: a soft component at $T_{s} \simeq(2-5) 10^{6} \mathrm{~K}$ and a hard component at $T_{h} \simeq(1.5-3) 10^{7} \mathrm{~K}$ (see Glassgold et al. 2000 for a recent review). The hard component is likely related to the nonstationary flaring activity (T. Montmerle 2002, private communication). Hence, we have modeled the circumstellar radiation field with optically thin, thermal bremsstrahlung emission from purely hydrogenic gas. Two components, soft and hard, at $3.5 \times 10^{6}$ and $2.8 \times 10^{7} \mathrm{~K}$, respectively, have been considered. ${ }^{13} \mathrm{~A}$ total X-ray luminosity of $3 \times 10^{29} \mathrm{ergs} \mathrm{s}^{-1}$ is assumed. We have simplified the geometry and assumed that the $\mathrm{X}$-ray source is pointlike and located in the center of a cylinder with radius $\log R_{b}=11.6$ and thickness $\log \Delta R=10.6$. A set of calculations with different belt densities $\left(10^{9}-10^{12} \mathrm{~cm}^{-3}\right)$ have been run. As expected, the results show that the FUV line emission is concentrated in an inner thin layer and that the emissivity is about 4 orders of magnitude smaller than that derived for the collisional plasma. Only at high densities $\left(10^{11} \mathrm{~cm}^{-3}\right)$ does $\mathrm{Si}$ III $]_{1892}$ emissivity become comparable to $\mathrm{C}$ III $]_{1908}$ emissivity, but the $\left.\mathrm{O}{ }_{\mathrm{III}}\right]_{1665}$ emissivity remains always $2-3$ orders of magnitude smaller than the Si III $]_{1892}$ and C III] $]_{1908}$ lines. Therefore, it seems unfeasible that this mechanism is the major source of the FUV line excitation in the RW Aur environment.

\footnotetext{
${ }^{11} V=\left[F\left(\mathrm{C}_{\text {III }}\right) 10^{1.03 A_{V}} 4 \pi d^{2}\right] / \epsilon_{1908}$, where $F\left(\mathrm{C}_{\text {III }}\right) 10^{1.03 A_{V}}$ is the detected line flux corrected from extinction and $\epsilon_{1908}$ is the line emissivity. $A_{V}=0.5$ (P2001) and the interstellar extinction law have been adopted to calculate the extinction at UV wavelengths.

${ }^{12}$ For instance, if the emission was produced in a torus with section $\pi r_{t}^{2}$, we derive that $r_{t}=1.9 \times 10^{8} \mathrm{~cm}$ for a fiducial belt radius of $4.4 R_{*}$, which is 2 orders of magnitude smaller than the radius of the belt.

${ }^{13}$ We have introduced both the soft and hard components in the photoionizing spectrum to analyze the physical conditions when submitted to the hardest X-ray's flux.
} 
Local photoionization by nearby reconnection events.Frequent reconnection events are expected to occur in the ionized belt due to the interaction with the stellar and accretion disk magnetic fields. The magnetic energy released in these events will be radiated in the X-ray range, photoionizing the nearby gas. The topology of the field may be very complex and the filling factor of the emitting regions small, as suggested above. We have assumed that the FUV line emission is produced in spherical gas cloudlets around small reconnecting loops $\left(R_{\text {loop }}=10^{8} \mathrm{~cm}^{-3}\right)$ that are located within the belt. We have modeled the radiation of the reconnecting loops as thermal bremstrahlung emission from hydrogenic gas. A luminosity of $10^{27} \mathrm{ergs} \mathrm{s}^{-1}$ is assumed. Two grids of models with fiducial temperatures $T_{e}=10^{6}$ and $T_{e}=10^{7} \mathrm{~K}$ have been run. The calculations have been carried for cloudlet densities from $10^{9}$ to $10^{12} \mathrm{~cm}^{-3}$. As shown in Figure 10, if the density is $\leq 10^{9} \mathrm{~cm}^{-3}$, the $\mathrm{C}$ IIII $]_{1908}$ emissivity is always larger than the $\mathrm{Si}$ III] $]_{1892}$ emissivity, which is not observed in RW Aur. For $n=10^{10} \mathrm{~cm}^{-3}$, both emissivities may be comparable, but the $\mathrm{O}$ III $]_{1665}$ emissivity is still small. Only at $n \geq 10^{11} \mathrm{~cm}^{-3}$ do the three FUV lines have comparable emissivities. Therefore, the FUV line ratios can be reproduced with this mechanism. The derived density of the plasma is similar to that calculated from collisional models, although the temperature is significantly smaller $\left(\log T_{e} \simeq 4.3\right)$.

We conclude that the belt FUV emission is produced in a high-density environment with $n \simeq 10^{11} \mathrm{~cm}^{-3}$. In fact, this environment is probably quite similar to the one observed in the solar transition region, where both collisional excitation and photoionization by X-ray radiation are involved in line excitation. In such an environment, the interpretation of the He I lines is very difficult (see Milkey 1975; Andretta \& Jones 1997; Macpherson \& Jordan 1999).
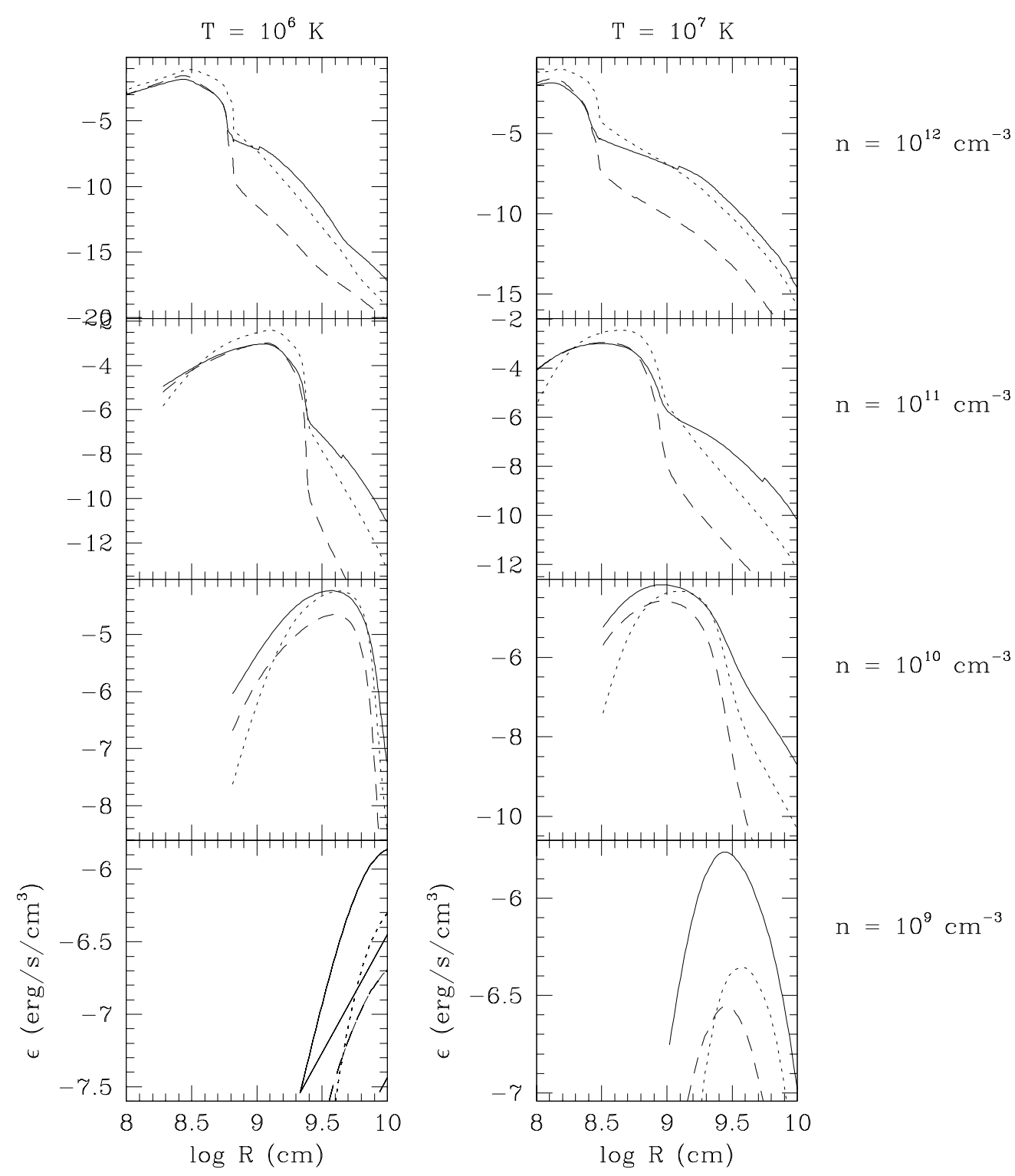

FIG. 10.-Emissivity of the $\mathrm{C}$ III $]_{1908}$ (solid line), $\mathrm{Si}$ III $]_{1892}$ (dotted line), and $\mathrm{O}$ III] $]_{1665}$ (dashed line) lines from spherical cloudlets around small reconnecting loops $\left(R_{\text {loop }}=10^{8} \mathrm{~cm}^{-3}\right)$. Loop radiation is assumed to be thermal bremstrahlung emission from hydrogenic gas at $T_{e}=10^{6} \mathrm{~K}($ left $)$ and $T_{e}=10^{7} \mathrm{~K}($ right $)$. Cloudlet densities, $n$, range from $10^{9}$ to $10^{12} \mathrm{~cm}^{-3}$. 


\section{CONCLUSIONS}

In summary, this work provides evidence of the existence of an ion belt within the stellar magnetosphere of RW Aur. Given the size and luminosity, the belt is going to contribute significantly to the optical veiling and probably traces an accretion-fed structure independent of the magnetic funnels. The study of RW Aur suggests that both accretion structures, magnetic funnels and ion belts, coexist.

The HST observations (UV and optical) also show that there are very short timescale variations in RW Aur in timescales significantly shorter than the 2.77 days reported by Gahm et al (1999). The interaction between the stellar magnetosphere and the disk may drive oscillations as well as magnetic precession of the inner disk (or the corotating belt) with characteristic timescales of $\sim 1$ day (Lai 1999). This points out that to get further insight into the RW Aur variability puzzle brilliantly described in P2001, a shortterm monitoring of this star is required. High spatial resolution is instrumental to avoid averaging out the variations in the complex circumstellar environment.

We thank T. Ray and F. Bacciotti for making us aware of some characteristics of the STIS optical observations. We also thank J. Valenti for useful comments on the HST STIS performance. This research was partially supported by the MCyT of Spain through grant AYA2000-966.

\author{
Andretta, V., \& Jones, H. P. 1997, ApJ, 489, 375 \\ Bastien, P. 1982, A\&AS, 48, 153 \\ Beristain, G., Edwards, S., \& Kwan, J. 2001, ApJ, 551, 1037 \\ Brandt, J. C., et al. 2001, AJ, 121,2173 \\ Dougados, C., Cabrit, S., Lavalley, C., \& Ménard, F. 2000, A\&A, 357, L61 \\ Ferland, G. J. 1996, HAZY: A Brief Introduction to CLOUDY \\ (Lexington: Univ. Kentucky) \\ Gahm, G. F., Petrov, P. P., Duemmler, R., Gameiro, J. F., \& Lago, \\ M. T. V. T. 1999, A\&A, 352, L95 \\ Ghez, A. M., White, R. J., \& Simon, R. 1997, ApJ, 490, 353 \\ Glassgold, A. E., Feigelson, E. D., \& Montmerle, T. 2000, Protostars and \\ Planets IV, ed. V. Manning, A. P. Boss, \& S. S. Russell (Tucson: Univ. \\ Arizona Press), 429 \\ Gómez de Castro, A. I. 2002, MNRAS, 332, 409 \\ Gómez de Castro, A. I., \& Franqueira, M. 1997, ULDA Access Guide to \\ T Tauri Stars Observed with IUE (ESA-SP 1205; Noordwijk: ESA) \\ Gómez de Castro, A. I., \& Lamzin, S. A. 1999, MNRAS, 304, L41 \\ Gómez de Castro, A. I., \& Verdugo, E. 2001, ApJ, 548, 976
}

\section{REFERENCES}

Gómez de Castro, A. I., \& Verdugo, E. 2002, Highlights of Spanish Astrophysics, (Dordrecht: Kluwer), in press

Grinin, V. P., Petrov, P. P., \& Shakhovskaya, N. I. 1983, Activity in Red Dwarfs Stars, ed. B. P. Byrne \& M. Rodonó (Dordrecht: Reidel), 513

Haman, F. 1994, ApJS, 93, 485

Haman, F., \& Persson, S. E. 1992, ApJS, 82, 247

Hirth, G. A., Mundt, R., \& Solf, J. 1997, A\&AS, 126, 437

Hirth, G. A., Mundt, R., Solf, J., \& Ray, T. P. 1994, ApJ, 427, L99

Johns, C. M., \& Basri, G. 1995, AJ, 109, 2800

Lai, D. 1999, ApJ, 524, 1030

López-Martín, L., Cabrit, S., \& Dougados, C. 2003, A\&A, 405, L1

Macpherson, K. P., \& Jordan, C. 1999, MNRAS, 308, 510

Milkey, R. W. 1975, ApJ, 199, L131

Petrov, P. P., Gahm, G. F., Gameiro, J. F., Duemmler, R., Ilyin, I. V., Laakkonen, T., Lago, M. T. V. T., \& Tuominen, I. 2001, A\&A, 369, 993 Stout-Batalha, N. M., Batalha, C. C., \& Basri, G. S. 2000, ApJ, 532, 474

Woitas, J., Ray, T. P., Bacciotti, F., Davis, C. J., \& Eisloeffel, J. 2002, ApJ, 580,336 